Pacific Journal of Mathematics

VERSUM SEQUENCES IN THE BINARY SYSTEM 


\title{
VERSUM SEQUENCES IN THE BINARY SYSTEM
}

\author{
Charles W. Trigg
}

Let the reverse of any positive integer $N$ be $N^{\prime}$ and $N+N^{\prime}=S_{1}$. Then

$$
S_{1}+S_{1}{ }^{\prime}=S_{2}, S_{2}+S_{2}{ }^{\prime}=S_{3}, \cdots, S_{k-1}+S_{k-1}{ }^{\prime}=S_{k} .
$$

Each of the $S_{i}$, the result of a reversal-addition operation on an integer, can appropriately be called a versum (a term coined in 1965 by Michael T. Rebmann and Frederick Groat while undergraduates at Carleton College). Thus, reiteration of the operation generates a sequence of versums. An ancient conjecture says that for every $N$ there is a palindromic $S_{k}$. True for one-digit and two-digit $N$ 's in the decimal system, considerable doubt has been thrown upon the universal verity of the conjecture $[1,2]$.

In the binary system, $D$. C. Duncan disproved the conjecture by a counter-example in which the sequence exhibits a palindrome-free recursive cycle of four versums (hereafter referred to as a PFRC-4). Sprague, Gabai and Coogan, and Brousseau rediscovered the same cycle. Brousseau also found two other palindrome-free sequences, each with a different PFRC-4.

Duncan remarked that "it would be highly interesting to establish the existence of numbers that neither become palindromic nor show a periodic recursion of cycles of digits." Such integers are reported below, together with an unlimited number of distinct palindrome-free recursive cycles in the binary system.

In the following discussion, all $N$ 's and $S$ 's are expressed in the binary system. All other numbers, including subscripts, exponents, and ratios, are expressed in the decimal system. Integers containing long sequences of the same digit are expressed compactly by replacing such a sequence by a single digit followed by a subscript indicating the number of like digits in that sequence. Thus,

$$
1011111010000 \equiv 101_{5} 010_{4} \text {. }
$$

Basic integers. The nature of the versum sequences of all integers in a particular scale of notation may be determined from the sequences of basic integers. A basic integer is the smallest member of the family of integers, all of which have the same $S_{1}$. For example:

$$
S_{1}(100110)=S_{1}(101010)=S_{1}(110100)=S_{1}(111000)=111111 .
$$


In the binary scale there are 1 one-digit and 2 two-digit basic integers. In general, if there are $t$ basic integers with $2 n$ digits, there are $2 t$ with $2 n+1$ digits, and $3 t$ with $2 n+2$ digits. Consequently, there are 404 basic integers $\leqq 10^{10}(=1024)$.

Trees and trunks. Those basic integers which are not produced by a reversal-addition operation are called sequence starters. Since many basic integers (or members of their families) are produced by the operation, the number of sequences that need be examined is $<404$.

Furthermore, the $S_{i}$ of one sequence may equal the $S_{j}$ of another sequence, thus effecting a merger at that stage. Merged sequences are called trees.

The sequence in a tree which contains the greatest number of palindromes is called a trunk and its smallest member is the trunk starter. If two sequences in a tree contain the same greatest number of palindromes, the longer measured from their merger is designated as the trunk. However, should these two sequences have equal lengths, the one with the smaller starter is called the trunk.

The versum sequences derived from the 404 basic integers $\leqq 10^{10}$ merge into 17 trees which are identified by capital letters and discussed separately below. A portion of one branched branch of tree $A$ showing six mergers is given in Figure 1. For every $S_{i}$, the integer directly below it, or if none is there then the one to the left of it on the next line, is $S_{i+1}$.

\begin{tabular}{|c|c|c|c|c|}
\hline \\
\hline \multicolumn{5}{|l|}{$\begin{array}{r}1 \\
10\end{array}$} \\
\hline \multicolumn{5}{|l|}{11} \\
\hline 110 & \multirow{2}{*}{\multicolumn{4}{|c|}{1011}} \\
\hline 1001 & & & & \\
\hline 10010 & \multicolumn{3}{|c|}{11000} & \\
\hline 11011 & & & & \\
\hline 110110 & 1001000 & 110010 & 1000100 & \\
\hline 1010001 & & 1000101 & 1010101 & 1110101 \\
\hline 10010110 & & & 10101010 & 11001100 \\
\hline
\end{tabular}

FiguRe 1. A portion of tree A

The coincidence ratio. The coincidence ratio of an integer is the ratio of the number of coincidences between the digits of the integer and the digits of its reverse to the number of digits in the integer. This ratio may have values ranging from 0, as for 10110010 , through such as $3 / 5$ for 10011 , up to 1 , the ratio for all palindromes. 
Thus the coincidence ratio is a measure of the deviation of an integer from palindromic status.

For any versum sequence, the appearance of a repetitive cycle in the numerators of successive ratios indicates that a recursive cycle in the digits of successive versums soon will appear. Usually the versum pattern lags behind the numerator pattern by one or two operations. A numerator cycle in ratios $<1$ assures that no more palindromes will appear in that versum sequence.

The values of the coincidence ratio fluctuate widely. When the coincidence ratio is continuously plotted against the sequence number of the versum, after a repetitive cycle appears, the smooth upper and lower boundary curves of the fluctuating curve become asymptotic to the sequence number axis. In the absence of a repetitive cycle, convergence of the boundary curves proceeds more slowly, but continuance of the convergence upon a ratio value $<1$ virtually assures the continued absence of palindromes in the corresponding versum sequence.

The procedure. Trees involving the sequences belonging to all positive integers $\leqq 10^{10}$ were constructed. Their trunks were identified and extended to the 32-digit level. Using a program written by Robert S. Cook, the trunk starters were fed into a computer by Vincent Grannell at Los Angeles City College. The printout for each trunk contained each versum in the sequence, its coincidence ratio in proper fraction form, and the number of the operation which produced the versum. Palindromes were first identified by their ratios. When a recursive cycle was observed in the ratio numerators, the printouts were continued until the distinctiveness of the pattern of the recursive cycle in the versums of the trunk was established.

High level mergers reduced the trees to 17 . These have been grouped principally according to the recursive patterns of the coincidence ratio numerators and identified by capital letters. Their distinctive properties are discussed in the following sections.

If mergers occurred beyond the last palindrome in the trunk, in some cases the trunk recursive patterns could be traced back down a merged branch to a versum smaller than the merger versum.

In the one case that a recursive cycle in the ratio numerators had not appeared after 300 operations, a computer program was continued with the printout showing only the operation number, the ratio in proper fraction form, and the ratio in decimal fraction form.

The A tree. This tree, with the trunk starter $10^{8}$, is the most complicated one. It contains 113 starters and 57 palindromes. Small 
branches merge with larger branches. The last branch merger with the trunk is at the 52-digit level, where $S_{78}\left(10^{8}\right)=S_{68}(1000110)$. Some of the branches are longer than the trunk at the merger versum. Indeed, $S_{53}(1)=S_{38}\left(10^{8}\right)$, so the last merger versum of the trunk= $S_{93}(1)$.

The powers of 10 contained in this tree are $1,10,10^{2}, 10^{3}, 10^{5}$, $10^{7}, 10^{8}$, and $10^{10}$.

The $A$ trunk contains palindromes at $S_{1}, S_{3}, S_{5}, S_{7}, S_{11}$, and $S_{13}$. The sequence starting with $10^{10}$, which merges with the trunk at the 32-digit level, also contains 6 palindromes, and at the same operational levels.

The greatest number of operations necessary to reach a first palindrome from a starter is 8 , for

$$
S_{8}(1110111111)=S_{8}(1110010111)=1_{16} \text {. }
$$

The largest palindrome occurring in this tree is $S_{21}(1000110)=$ $10010_{4} 1011010_{4} 1001$. No palindrome appears beyond the 21st operational level.

A repetitive pattern, 5-5-4-4, appears in the numerators of the coincidence ratios of the trunk versums starting at $S_{36}$. A repetitive pattern in the versums begins with

$$
S_{38}=101_{x} 010_{x} \text {, where } x=14 .
$$

The next four versums of the sequence are

$$
\begin{aligned}
& 110_{x-2} 10001_{x-2} 01 \\
& 101_{x} 010_{x+1} \\
& 110_{x} 101_{x-1} 01 \\
& 101_{x+1} 010_{x+1} .
\end{aligned}
$$

Clearly, none of these versums is a palindrome. Thus all starters in the $A$ tree generate versum sequences that eventually lead to a PFRC-4 for $x>1$, and hence to an endless palindrome-free sequence.

52 of these starters, such as 10110, 100011, 1011101, 11000111, 110001010 , and 1000111000 , produce sequences which generate no palindromes, and which begin at various levels to conform to the four-term repetitive pattern. Indeed, the pattern extends back through connecting branches to $S_{4}(10110)$.

This is Duncan's [2] pattern.

It is further observed that an integer of the form $101_{x} 010_{y}$, with $y<x$, generates a two-term pattern in which $x$ stays constant and $y$ increases. When $y$ reaches equality with $x$, the sequence falls into Duncan's pattern. 
The B groups of trees. Four of the independent trunk sequences belong to a generic group in which the numerators of the coincidence ratios eventually fall into the repetitive pattern, 8-8-11-11.

The trunk starter of the $B_{1}$ tree is 100001010 . There are only 3 other starters. The last merger is at $S_{4}$, the 12-digit level. The trunk palindromes are $S_{1}, S_{5}$, and $S_{7}$. These are the only palindromes in the tree. The repetitive pattern of the coincidence ratio numerators begins at $S_{9}$, the pattern of the versums at $S_{10}$.

The trunk and only starter of the $B_{2}$ tree is 100010011. The trunk palindromes are $S_{6}$ and $S_{8}$. The numerator pattern begins at $S_{29}$, the versum pattern at $S_{31}$.

The trunk starter of the $B_{3}$ tree is 1001010 . There are 3 other starters. The last merger is at $S_{4}$, the 10-digit level. The only palindromes are the trunk palindromes $S_{11}$ and $S_{13}$. The numerator pattern begins at $S_{30}$, the versum pattern at $S_{32}$.

The $B_{4}$ tree is palindrome-free. The trunk starter is 110111110 , the others are 110111010 and 1001111000. The last merger is at $S_{3}$, the 12-digit level. The numerator pattern begins at $S_{35}$, the versum pattern at $S_{38}$.

The most operations required in the $B$ group to produce a first palindrome are those to produce $S_{11}(1001010)$. The largest palindrome in the group is the 16-digit

$$
S_{13}(1001010)=1001101111011001 \text {. }
$$

Significant versums from each of the $B$ trunk sequences are:

$$
\begin{array}{ll}
B_{1}: & S_{10}(100001010)=101_{2} 010_{2} 101_{2} 010_{3} \\
B_{2}: & S_{33}(100010011)=101_{6} 010_{4} 101_{4} 010_{7} \\
B_{3}: & S_{34}(1001010)=101_{5} 010_{5} 101_{5} 010_{6} \\
B_{4}: & S_{36}(110111110)=101_{4} 010_{9} 101_{9} 010_{5} .
\end{array}
$$

Each of these has the generalized form

$$
101_{x} 010_{a} 101_{a} 010_{x+1} \text {. }
$$

The next four versums produced from this integer by repeated application of the reversal-addition operation are:

$$
\begin{aligned}
& 110_{x} 101_{a} 010_{a} 101_{x-1} 01 \\
& 101_{x+1} 010_{a} 101_{a} 010_{x+1} \\
& 110_{x-1} 10001_{a-2} 01110_{a-2} 10001_{x-1} 01 \\
& 101_{x+1} 010_{a} 101_{a} 010_{x+2} .
\end{aligned}
$$

None of these is a palindrome. The fifth versum has the same 
general form as the first versum, with the value of the integer $x$ increasing by 1 . Thus for each constant integer value of $a>1$ a versum sequence exists with a PFRC-4. Within this limitless set, the sequences for $a=2,4,5$, and 9 are the only ones generated from values of $N \leqq 10^{10}$. When $x=1$ and $a=2$, the first generalized form becomes a 15-digit integer, so the versum sequence in which it appears must derive form $N>10^{10}$.

The $\mathbf{C}$ group of trees. Five of the independent trunk sequences belong to a second generic group, since the numerators of the coincidence ratios of each of them eventually fall into the repetitive pattern, 9-9-10-10.

The lone starter of the $C_{1}$ tree is $1_{3} 0_{3} 1_{4}$. This trunk contains the two palindromes $S_{4}$ and $S_{6}$. The repetitive pattern in the numerators of the coincidence ratios begins at $S_{8}$, the repetitive pattern of the versums begins at $S_{9}$.

The trunk starter of the $C_{2}$ tree is $1_{3} 0_{3} 110$. There are 9 other starters. The last merger occurs at $S_{18}$ at the 23-digit level. The trunk palindromes are $S_{9}, S_{11}$, and $S_{13}$. There are 3 more palindromes in the tree. The numerator pattern begins at $S_{18}$, the versum pattern at $S_{18}$. Five starters, $1001_{4} 001,101_{6} 00,1001_{7}, 10_{3} 110_{4}$, and $10_{4} 11001$, in $C_{2}$ generate palindrome-free sequences. They all merge into the branch which merges with the trunk at $S_{18}$. Indeed, the PFRC-4 in $C_{2}$ starts in this branch at $S_{13}\left(1001_{4} 001\right)$.

There are 6 starters in the $C_{3}$ tree, the trunk starter being 10 100. The last merger is at $S_{6}$ at the 12-digit level. The only palindromes are the trunk palindromes $S_{1}, S_{3}, S_{11}, S_{13}, S_{15}, S_{19}$, and $S_{21}$. The numerator pattern begins at $S_{28}$, the versum pattern at $S_{30}$.

The 11 starters in the $C_{4}$ tree include the trunk starter $10_{6} 11$. The last merger is at $S_{25}$ at the 26-digit level. Of the 13 palindromes in the tree, 10 are in the trunk, namely: $S_{8}, S_{10}, S_{12}, S_{16}, S_{18}$, $S_{28}, S_{30}, S_{32}, S_{36}$, and $S_{38}$. The numerator pattern begins at $S_{63}$, the versum pattern at $S_{65}$. The sequence based on $10^{11}$ merges with this trunk at $S_{3}\left(10^{11}\right)=S_{8}\left(10_{6} 11\right)$. Since $S_{1}\left(10^{11}\right)$ is a palindrome, the sequence starting with $10^{11}$ contains the eleven palindromes $S_{1}, S_{3}, S_{5}, S_{7}, S_{11}, S_{13}$, $S_{23}, S_{25}, S_{27}, S_{31}$, and $S_{33}$.

The sole starter in the $C_{5}$ tree is $110_{3} 1_{3} 0$. This sequence contains the palindromes $S_{21}, S_{23}, S_{33}, S_{35}, S_{37}, S_{41}$ and $S_{43}$. The numerator pattern begins with $S_{76}$, the versum pattern with $S_{78}$.

In the $C$ group, the greatest number of operations needed to reach a first palindrome is that to form $S_{21}\left(110_{3} 1_{3} 0\right)$.

The largest palindrome occurring in any $C$ sequence is the 39digit $S_{43}\left(110_{3} 1_{3} 0\right)=101_{6} 010_{19} 101_{6} 01$. 
Significant versums from each of the $C$ trunk sequences are:

$$
\begin{aligned}
& C_{1}: S_{11}(1110001111)=101_{3} 010_{2} 101_{3} 010_{3} \\
& C_{2}: S_{18}(111000110)=101_{4} 010_{3} 101_{4} 010_{4} \\
& C_{3}: S_{30}(10000100)=101_{6} 010_{4} 101_{5} 010_{6} \\
& C_{4}: S_{67}(100000011)=101_{11} 010_{10} 101_{11} 010_{11} \\
& C_{5}: S_{80}(110001110)=101_{13} 010_{12} 101_{13} 010_{13} .
\end{aligned}
$$

Each of these versums has the generalized form

$$
101_{x} 010_{a} 101_{a+1} 010_{x} \text {. }
$$

The next four versums following this generalized integer are

$$
\begin{aligned}
& 110_{x-2} 10001_{a} 010_{a-1} 10001_{x-2} 01 \\
& 101_{x} 010_{a+1} 101_{a} 010_{x+1} \\
& 110_{x} 101_{a-1} 01110_{a} 101_{x-1} 01 \\
& 101_{x+1} 010_{a} 101_{a+1} 010_{x+1} .
\end{aligned}
$$

None of these is a palindrome, and the fifth versum has the form of the first versum with the variable $x$ increasing by 1 . Thus a second generic class of versum sequences containing a PFRC-4 is established in which $a>0$ is a constant, and $x>2$ steadily increases. In this unlimited class, the only sequences generated by integers $<10^{10}$ have $a=2,3,4,10$, and 12. When $a=1$ and $x=3$, the first generalized form becomes a 17-digit integer. For values of $a$ other than the indicated five, the versum sequences with PFRC-4's of this form must have starters $>10^{10}$.

The $\mathrm{D}$ tree. This tree has four starters including the trunk starter 10000 with mergers at $S_{5}$ and $S_{7}$. All tree palindromes are the trunk palindromes $S_{1}, S_{3}, S_{5}, S_{15}$, and $S_{19}=1101_{12} 011$. A repetitive numerator pattern, 22-22-27-27, starts at $S_{180}$ A PFRC-4 starts in the versum sequence at $S_{182}$ at the 123-digit level. Now,

$$
S_{184}=101_{x} 010_{a} 101_{b} 010_{c} 101_{d} 010_{d} 101_{c} 010_{b-1} 101_{a+1} 010_{x},
$$

where $x=12, a=11, b=9, c=2, d=18$.

The next four versums of the PFRC-4 based on this generalized form are

$$
\begin{aligned}
& 110_{x-2} 10_{3} 1_{a} 010_{b-2} 10_{3} 1_{c-2} 01_{3} 0_{d-2} 10_{3} 1_{d-2} 01_{3} 0_{c-2} 10_{3} 1_{b-1} 010_{a-1} 10_{3} 1_{x-2} 01 \\
& 101_{x} 010_{a+1} 101_{b-1} 010_{c} 101_{d} 010_{d} 101_{c} 010_{b} 101_{a} 010_{x+1} \\
& 110_{x} 101_{a-1} 01110_{b-1} 101_{c} 010_{d} 101_{d} 010_{c} 101_{b-2} 01110_{a} 101_{x-1} 01 \\
& 101_{x+1} 010_{a} 101_{b} 010_{c} 101_{d} 010_{d} 101_{c} 010_{b-1} 101_{a+1} 010_{x+1} .
\end{aligned}
$$


Thus the $D$ tree leads to an infinite class of PFRC-4 versum sequences with the variable $x>2$, and four constants: $a>1, b>1, c>1$, and $d>1$.

The $\mathrm{E}$ tree. The $E$ tree has 11 starters including the trunk starter $10_{5} 1100$. Mergers occur at $S_{3}$ and $S_{6}$. All tree palindromes are the trunk palindromes $S_{1}, S_{11}$, and $S_{18}$ which has 18 digits.

A repetitive pattern, 26-26-23-23, in the numerators of the coincidence ratios starts at $S_{47}$. The repetitive pattern of the versums starts with $S_{48}$. Now,

$$
S_{50}=101_{x} 010_{a} 101_{b} 010_{c} 101_{d} 010_{d+1} 101_{c} 010_{b-1} 101_{a+1} 010_{x},
$$

where $x=4, a=3, b=2, c=2$, and $d=1$.

The next four versums of the sequence containing this general form are

$$
\begin{aligned}
& 110_{x-2} 10_{3} 1_{a} 010_{b-2} 10_{3} 1_{c-2} 01_{3} 0_{d} 101_{d-1} 01_{3} 0_{c-2} 10_{3} 1_{b-1} 010_{a-1} 10_{3} 1_{x-2} 01 \\
& 101_{x} 010_{a+1} 101_{b-1} 010_{c} 101_{d+1} 010_{d} 101_{c} 010_{b} 101_{a} 010_{x+1} \\
& 110_{x} 101_{a-1} 01110_{b-1} 101_{c} 010_{d-1} 10001_{d} 010_{c} 101_{b-2} 01110_{a} 101_{x-1} 01 \\
& 101_{x+1} 010_{a} 101_{b} 010_{c} 101_{d} 010_{d+1} 101_{c} 010_{b-1} 101_{a+1} 010_{x+1} .
\end{aligned}
$$

Thus the $E$ tree sequence leads to another infinite class of PFRC-4 versum sequences with variable $x>2$, and four constants: $a>1$, $b>1, c>1$, and $d>0$.

The $\mathrm{F}$ tree. The $F$ tree has five starters including the trunk starter $10_{5} 1010$, all merging at $S_{3}$. All palindromes are the trunk palindromes $S_{1}, S_{33}$, and the 80-digit

$$
S_{113}=10010_{25} 10010010101101010010010_{25} 1001 \text {. }
$$

A recurrent pattern of the coincidence ratio numerators, 10-15-13-14, starts at $S_{163}$. A PFRC-4 starts in the versums with

$$
S_{164}=101_{x} 010_{a+4} 1_{b} 01_{a+2} 010_{x+1}
$$

where $x=14, a=30$, and $b=7$. Proceeding from the generalized form, the next four versums are

$$
\begin{aligned}
& 110_{x} 101_{a+2} 001_{b-3} 01110_{a+2} 101_{x-1} 01 \\
& 101_{x+1} 010_{a+1} 10001_{b-4} 00001_{a+2} 010_{x+1} \\
& 110_{x-1} 10001_{a} 0101010100110_{a} 10001_{x-1} 01 \\
& 101_{x+1} 010_{a+4} 1_{b} 01_{a+2} 010_{x+2} .
\end{aligned}
$$

Thus the $F$ trunk is a member of another infinite class of PFRC-4 
versum sequences with variable $x>1$, and two constants: $a>0$ and $b>6$.

The $G$ tree. The $G$ tree contains three starters including the trunk starter 1100011010 . The 3 sequences merge at $S_{2}$. The only palindrome is the 24-digit,

$$
S_{19}(1100011010)=100100101_{8} 01001001 \text {. }
$$

A recurrent pattern, 17-21-26-22, in the numerators of the coincidence ratios starts at $S_{254}$. The PFRC-4 of the versums starts at the 174digit level with $S_{256}=101_{x} 010_{a} 101_{b} 010110_{c} 101_{c} 010110_{b+1} 101_{a} 010_{x}$ where $x=15, a=19, b=20$, and $c=22$. Proceeding from the generalized form the next four versums are

$$
\begin{aligned}
& 110_{x-2} 10_{3} 1_{a-2} 01_{3} 0_{b-1} 10_{5} 1_{c} 010_{c-1} 10_{5} 1_{b-1} 01_{3} 0_{a-2} 10_{3} 1_{x-2} 01 \\
& 101_{x} 01_{a} 101_{b+1} 0_{3} 10_{c+1} 101_{c} 0_{3} 10_{b+1} 101_{a} 010_{x+1} \\
& 110_{x} 101_{a} 010_{b+3} 101_{c-1} 01_{3} 0_{c+2} 101_{b+1} 010_{a} 101_{x-1} 01 \\
& 101_{x+1} 010_{a} 101_{b} 010110_{c} 101_{c} 010110_{b+1} 101_{a} 010_{x+1} .
\end{aligned}
$$

Thus the $G$ tree leads to another infinite class of PFRC-4 versum sequences with variable $x>1$, and three constants: $a>1, b>0$, and $c>0$.

The $\mathbf{H}$ group of trees. The trunk starter of the $H_{1}$ tree is $10^{6}$. The sequences of the other 29 starters in the tree merge into five branches which merge with the trunk at $S_{5}, S_{7}, S_{9}, S_{12}$, and $S_{28}$. Of the 8 palindromes in the tree, 4 are in the trunk, namely: $S_{1}, S_{3}, S_{5}$, and $S_{7}=11011011011$.

The repetitive pattern, $5-4-6-7$, in the numerators of the coincidence ratios starts at $S_{11}$. The PFRC-4 of the versums starts with $S_{12}=101_{k} 010110_{k+1}$ where $k=4$. The next four versums based on this general form are

$$
\begin{aligned}
& 110_{k-1} 10_{5} 1_{k-1} 01 \\
& 101_{k+1} 00010_{k+2} \\
& 110_{k+3} 101_{k} 01 \\
& 101_{k+1} 010110_{k+2}
\end{aligned}
$$

None of these is palindromic. The pattern extends back into a branch to $S_{3}(1001101)$, where $k=1$. This is Brousseau's [1] second pattern.

The fifteen branch starters of palindrome-free sequences that merge with the trunk beyond its $S_{7}$ are: 1001101, 10 $11,10101_{3} 0$, $1010_{3} 100,10_{3} 110_{3}, 101101_{4}, 101010100,1001001_{3} 0,1101_{3} 011,1101_{6}$, $110_{3} 10010,1100110010,10_{4} 10_{3} 1,10_{3} 1_{6}$, and $1001_{5} 00$. 
The trunk starter of the $H_{2}$ tree is $10_{5} 100$. There are six branch starters. The last merger is at $S_{9}$ at the 15-digit level. The only palindromes are the trunk palindromes $S_{1}, S_{3}$, and $S_{5}=101101101101$. The numerator pattern, $5-9-8-8$, begins at $S_{8}$, the versum repetitive pattern at $S_{9}=110_{k} 1000100001_{k} 01$, where $k=1$. The next four versums based on this generalized form are

$$
\begin{aligned}
& 101_{k+2} 00100010_{k+3} \\
& 110_{k+4} 110101_{k+1} 01 \\
& 101_{k+2} 011100110_{k+3} \\
& 110_{k+1} 1000100001_{k+1} 01 .
\end{aligned}
$$

This is Brousseau's [1] third pattern. The sequence based on branch starter $110_{5} 111$ merges at $S_{9}$ and is palindrome-free.

The $H_{1}$ and $H_{2}$ trunks have different numerator patterns, but they are the first two members of an infinite class of PFRC-4 versum sequences with numerator repetitive patterns $5+3 a, 4+a, 6+3 a$, and $7+a$. With $(110)_{a}$ indicating that 110 is repeated $a$ times, consider the generalization

$$
110_{x+3}(110)_{a} 101_{x} 01, \text { where } x>0, a \geqq 0 .
$$

The next four versums based on this general form are:

$$
\begin{aligned}
& 101_{x+1} 01(110)_{a} 0110_{x+2} \\
& 110_{x} 1000(100)_{a} 001_{x} 01 \\
& 101_{x+2}(001)_{a} 00010_{x+3} \\
& 110_{x+4}(110)_{a} 101_{x+1} 01 .
\end{aligned}
$$

When $a=0$, this becomes the PFRC-4 of $H_{1}$, and when $a=1$, the PFRC-4 of $H_{2}$.

The $U$ tree. The $U$ tree has $10^{\circ}$ as a trunk starter, and two branch starters. The last merger is at $S_{3}$. The only palindromes in the tree are the trunk palindromes, $S_{1}, S_{3}, S_{5}, S_{7}, S_{11}, S_{29}$, and the 30-digit $S_{31}=10010_{8} 1011010_{8} 1001$. This sequence has been extended to $S_{6005}$, a 3433-digit integer. No pattern in the coincidence ratio numerators or in the versums has been detected. The ordinates of the smooth boundary curve passing through peak maximum values of the ratios drop from 1.000 to 0.662 . The ordinates of the smooth boundary curve for the minimum values increase from 0.059 to 0.304 .

The steady but slow decrease of the peak maximum ratios indicates that the sequence always will remain palindrome free. It would appear that this sequence will satisfy Duncan's [2] expressed interest in one that is palindrome-free but has no periodic recursion of cycles 
of digits.

Further generalizations. One of the versums in the PFRC-4 of tree $A$ is $101_{x} 010_{x}$. One of the versums in the generalized PFRC-4 of group $B$ is $101_{x} 010_{a} 101_{a} 010_{x}$. These are the simplest members of a larger generic group.

Let $V^{(x)}=101_{x}$ and $W^{(x)}=010_{x}$ be complementary modules. Any integer, composed of complementary pairs symmetrical to its center and with the $V$ 's and $W$ 's alternating, is part of a PFRC-4. That is, part of a PFRC-4 is

$$
V^{(x)} W^{\left(a_{1}\right)} V^{\left(a_{2}\right)} W^{\left(a_{3}\right)} \cdots V^{\left(a_{3}\right)} W^{\left(a_{2}\right)} V^{\left(a_{1}\right)} W^{(x)},
$$

where $x>1, a_{i}>1$. An example of this type of integer is

$$
101_{2} 010_{7} 101_{3} 010_{4} 101_{19} 010_{19} 101_{4} 010_{3} 101_{7} 010_{2} \text {. }
$$

Under the reiterated reversal addition operation, in every fourth versum the inner modules return in unaltered condition and in the two end modules $x$ increases by 1 .

A special case of this generalization consists of the repetition of the aforesaid $A$ trunk versum to form new integers. Indeed, if an integer $M=V^{(x)} Y W^{(x)}$ is a member of a PFRC-4, then $M_{y}$ and $V^{(x)} Y_{y} W^{(x)}$, with $y>0$, also are members of PFRC-4's. (As with repeated digits, the subscript $y$ indicates that $M$ and $Y$ are repeated $y$ times.) These extensions apply to the appropriate versums in the PFRC-4's of the trunk sequences in $A, B, C, D, E, F$, and $G$.

\section{Summary and observations.}

1. In the binary system, all versum sequences based on integers $<10^{10}$ merge into 17 trees. No merger occurs beyond any $S_{93}$ nor beyond the 52-digit level of the versums.

2. The greatest number of operations necessary to produce a first palindrome from any starter is that required for $S_{21}\left(110_{3} 1_{3} 0\right)$. However, within one of the sequences more operations are needed to produce the first palindrome of a non-starter, namely, for

$$
S_{80}\left[S_{33}\left(10_{5} 1010\right)\right] \text {. }
$$

3. The largest palindrome occurring in the 17 trunk sequences, and the one most remote from its starter is the 80 -digit $S_{113}\left(10_{5} 1010\right)$. Thus, in no sequence involved in this study does any palindrome occur beyond the 113 th operation or the 80-digit level.

4. The most palindromes in any versum sequence starting with $N<10^{10}$ are the ten in the sequence starting with $10_{6} 11$. However, eleven palindromes appear in the sequence starting with $10^{11}$. 
5. A recursive cycle appearing in the numerators of the coincidence ratios of the versums indicates the early occurrence of a recursive cycle in the versums.

6. All recursive cycles found in this study are cycles of four. On the basis of identity or similarity of the numerator cycles, 16 of the trunk sequence fall into 8 categories. Each category, except $A$, contains an infinity of sequences of versums with distinct PFRC-4's. These infinities may be called vertical. Expansion of the investigation to $N>10^{10}$ probably will reveal other infinite categories.

7. The latest appearance of a PFRC-4 in any versum sequence is by the one beginning with $S_{256}\left(110_{3} 11010\right)$.

8. In the categories $A, B, C, D, E, F$, and $G$ one term of each distinct PFRC-4 mentioned in 6 may be expanded horizontally by repetition in two ways to produce an infinity of new PFRC-4's. Also, by horizontal interpolation in the leading term of the $A$ tree PFRC-4, an infinity of new PFRC-4's can be formed.

9. The $U$ trunk sequence, with a last palindrome at $S_{31}$, was carried up to $S_{6005}$ without developing a recursive pattern in either the coincidence ratios or in the versums.

10. There are 80 basic starters, $\leqq 10^{10}$, representing 195 integers, which generate versum sequences which contain no palindromes.

\section{Conjectures.}

1. In the versum sequence developed from any integer in the binary system, the number of palindromes is finite. Consequently, every such sequence eventually becomes and remains palindrome-free.

2. There is an infinity of palindrome-free versum sequences in the binary system which exhibit no recursive cycle in the digits of the versums.

3. For every integer $k$, there is an $N$ in the binary system for which $S_{k}$ is a first palindrome.

$$
\left\{S_{80}\left[S_{33}\left(10_{5} 1010\right)\right] \text { establishes this for } k<81 .\right\}
$$

\section{REFERENCES}

1. Brother Alfred Brousseau, Palindromes by Addition in Base Two, Mathematics Magazine, 42 (November, 1969), 254-256.

2. D. C. Duncan, Sujet d'etude $n^{\circ}$ 74, Sphinx (Bruxelles), 9 (June, 1939), 91-92.

3. Hyman Gabai and Daniel Coogan, On Palindromes and Palindromic Primes, Mathematics Magazine, 42 (November, 1969), 252-254.

4. Roland Sprague, Recreation in Mathematics, Dover Publications Inc., (1963), Problem

5 , pages 6-7, 28-29.

5. Charles W. Trigg, Palindromes by Addition, Mathematics Magazine, 40 (January, 1967), 26-28. 
6. Charles W. Trigg, More on Palindromes by Reversal-Addition, Mathematics Magazine, 45 (September 1972), 184-186.

Received May 16, 1972.

2404 Loring St., San Diego, California 



\section{PACIFIC JOURNAL OF MATHEMATICS}

\section{EDITORS}

D. Gilbarg and J. Milgram

Stanford University

Stanford, California 94305

R. A. Beaumont

University of Washington

Seattle, Washington 98105
J. DUGUNDJI* Department of Mathematics

University of Southern California Los Angeles, California 90007

RICHARD ARENS

University of California

Los Angeles, California 90024

\section{ASSOCIATE EDITORS}

E. F. BeCKenbach

B. H. NeUMaNN

F. WOLF

K. Yoshida

\section{SUPPORTING INSTITUTIONS}

UNIVERSITY OF BRITISH COLUMBIA

UNIVERSITY OF SOUTHERN CALIFORNIA

CALIFORNIA INSTITUTE OF TECHNOLOGY

UNIVERSITY OF CALIFORNIA

MONTANA STATE UNIVERSITY

STANFORD UNIVERSITY

UNIVERSITY OF TOKYO

UNIVERSITY OF NEVADA

UNIVERSITY OF UTAH

NEW MEXICO STATE UNIVERSITY

WASHINGTON STATE UNIVERSITY

OREGON STATE UNIVERSITY

UNIVERSITY OF OREGON

OSAKA UNIVERSITY

UNIVERSITY OF WASHINGTON

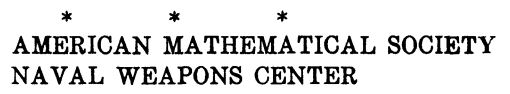

* C. DePrima will replace J. Dugundji until August 1974. 


\section{Pacific Journal of Mathematics}

\section{Vol. 47, No. $1 \quad$ January, 1973}

K. Adachi, Masuo Suzuki and M. Yoshida, Continuation of holomorphic

mappings, with values in a complex Lie group ....................

Michael Aschbacher, A characterization of the unitary and symplectic groups

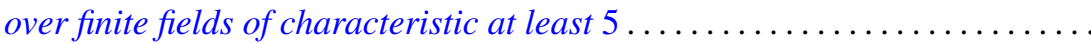

Larry Eugene Bobisud and James Calvert, Energy bounds and virial theorems for abstract wave equations....................................

Christer Borell, A note on an inequality for rearrangements ................

Peter Southcott Bullen and S. N. Mukhopadhyay, Peano derivatives and general

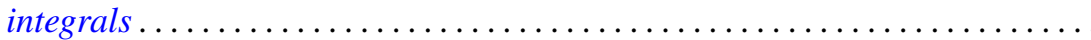

Wendell Dan Curtis, Yu-Lee Lee and Forrest Miller, A class of infinite dimensional subgroups of $\operatorname{Diff}^{r}(X)$ which are Banach Lie groups .........

Paul C. Eklof, The structure of ultraproducts of abelian groups ...............

William Alan Feldman, Axioms of countability and the algebra $C(X) \ldots \ldots \ldots$

Jack Tilden Goodykoontz, Jr., Aposyndetic properties of hyperspaces...........

George Grätzer and J. Płonka, On the number of polynomials of an idempotent algebra. II ...........................................

Alan Trinler Huckleberry, The weak envelope of holomorphy for algebras of

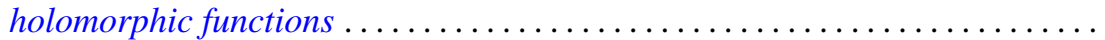

John Joseph Hutchinson and Julius Martin Zelmanowitz, Subdirect sum decompositions of endomorphism rings . . . . . . . . . . . . . . . .

Gary Douglas Jones, An asymptotic property of solutions of

$y^{\prime \prime \prime}+p y^{\prime}+q y=0$.

Howard E. Lacey, On the classification of Lindenstrauss spaces .

Charles Dwight Lahr, Approximate identities for convolution measure algebras.

George William Luna, Subdifferentials of convex functions on Banach

spaces.

Nelson Groh Markley, Locally circular minimal sets. .

Robert Wilmer Miller, Endomorphism rings of finitely generated projective modules

Donald Steven Passman, On the semisimplicity of group rings of linear

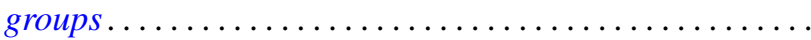

Bennie Jake Pearson, Dendritic compactifications of certain dendritic spaces.

Ryōtarō Satō, Abel-ergodic theorems for subsequences ...... .

Henry S. Sharp, Jr., Locally complete graphs. . .

Harris Samuel Shultz, A very weak topology for the Mikusinski field of

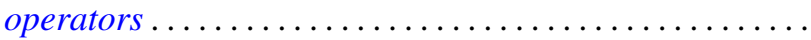

Elena Stroescu, Isometric dilations of contractions on Banach spaces ...

Charles W. Trigg, Versum sequences in the binary system ... . .

William L. Voxman, On the countable union of cellular decompositions of n-manifolds 